\title{
Evaluation of CERS2 Gene as a Potential Biomarker for Bladder Cancer
}

\author{
Ahmed Faris Aldoghachi, ${ }^{1}$ Aminuddin Baharudin $\left(\mathbb{D},{ }^{1}\right.$ Umar Ahmad $\left(D,{ }^{1}\right.$ Soon Choy Chan, ${ }^{2}$ \\ Teng Aik Ong, ${ }^{3}$ Rosna Yunus, ${ }^{4}$ Azad Hassan Razack, ${ }^{3}$ Khatijah Yusoff $\odot$, ${ }^{5,6}$ \\ and Abhi Veerakumarasivam $\left(\mathbb{D}^{1,6,7}\right.$ \\ ${ }^{1}$ Medical Genetics Laboratory, Faculty of Medicine and Health Sciences, Universiti Putra Malaysia, Selangor Darul Ehsan, Malaysia \\ ${ }^{2}$ Perdana University School of Foundation Studies, Perdana University, Selangor Darul Ehsan, Malaysia \\ ${ }^{3}$ Department of Surgery, Faculty of Medicine, University of Malaya, Wilayah Persekutuan, Kuala Lumpur, Malaysia \\ ${ }^{4}$ Histopathology Unit, Department of Pathology, Hospital Kuala Lumpur, Wilayah Persekutuan, Kuala Lumpur, Malaysia \\ ${ }^{5}$ Department of Microbiology, Faculty of Biotechnology and Biomolecular Sciences, Universiti Putra Malaysia, \\ Selangor Darul Ehsan, Malaysia \\ ${ }^{6}$ Malaysia Genome Institute, National Institute of Biotechnology Malaysia, Selangor Darul Ehsan, Malaysia \\ ${ }^{7}$ Department of Biological Sciences, School of Science and Technology, Sunway University, Selangor Darul Ehsan, Malaysia
}

Correspondence should be addressed to Abhi Veerakumarasivam; abhiv@sunway.edu.my

Received 16 November 2018; Revised 11 February 2019; Accepted 26 February 2019; Published 15 September 2019

Academic Editor: Tilman Todenhöfer

Copyright (C) 2019 Ahmed Faris Aldoghachi et al. This is an open access article distributed under the Creative Commons Attribution License, which permits unrestricted use, distribution, and reproduction in any medium, provided the original work is properly cited.

\begin{abstract}
The ceramide synthase 2 (CERS2) gene has been linked to tumour recurrence and invasion in many different types of cancers including bladder cancer. In this study, the expression levels of CERS2 in bladder cancer cell lines were analysed using qRT-PCR and the protein expression in clinical bladder cancer histopathological specimens were examined via immunohistochemistry. The potential utility of CERS2 as a predictive biomarker of response to oncolytic virotherapy was assessed by correlating the CERS2 mRNA expression to $\mathrm{IC}_{50}$ values of cells treated with the Newcastle disease virus (NDV), AF2240 strain. This study demonstrates that CERS2 is differentially expressed in different types of bladder cancer cell lines and that the siRNA-mediated downregulation of the expression of CERS2 reduces the migratory potential of UMUC1 bladder cancer cells. However, there were no significant correlations between the expression levels of the CERS2 protein with bladder cancer grade/stage or between the IC50 values of cells treated with NDV and CERS2 expression. Although the utility of CERS2 expression may be limited, its potential as an antimigration cancer therapeutic should be further examined.
\end{abstract}

\section{Introduction}

Bladder cancer is ranked as the ninth most common cancer worldwide, and men are more than three times more likely to develop the disease as compared to women $[1,2]$. Due to its high rate of recurrence and invasion, it has the highest cost of treatment per patient as compared to other malignancies [3]. Bladder cancer can be divided into two categories, nonmuscle-invasive bladder cancer and muscle-invasive bladder cancer. Nonmuscle-invasive bladder cancers account for $70 \%$ of all bladder cancers and are associated with low risk of progression and metastasis but high risk of recurrence. The treatment of nonmuscle invasive bladder cancer includes transurethral resection followed by a course of chemotherapy or immunotherapy to reduce the recurrence of the tumour $[4,5]$. In contrast, muscle-invasive bladder cancers are associated with high rates of progression and metastasis and are usually treated by radical cystectomy if the tumour is organ-confined $[4,6]$. To date, there are no prognostic or diagnostic biomarkers as well as predictive biomarkers of therapeutic response that are currently used in the mainstream management of bladder cancer patients $[7,8]$. 
The CERS2 gene, also known as the tumour metastasis suppressor gene 1 (TMSG1), contains Hox-like and TLC domains and is involved in the synthesis of long acyl chain ceramides. This gene was found to be expressed in 12 different types of human tissues, with the highest expression in the liver and kidney and the lowest expression in the colon, spleen, small intestines, thymus, and peripheral blood leukocytes [9]. Increased mRNA levels of the CERS2 gene were observed in metastatic cell lines as compared to nonmetastatic cell lines, implicating a role for CERS2 in metastasis [10]. Other studies have identified a correlation between CERS2 expression with the degree of recurrence and invasion in liver cancer [11], breast cancer [12], cervical cancer [13], pancreatic cancer [14], ovarian cancer [14], prostate cancer [15], and bladder cancer [11]. In a study conducted by Wang et al. [11], bladder cancer patients with low CERS2-expressing tumours were shown to have a poorer clinical prognosis.

In recent years, the use of oncolytic viruses such as the Newcastle disease virus (NDV) has become an emerging cancer therapeutic modality. The idea of using NDV in the treatment of cancer has gained vast interest because these viruses are capable of selectively targeting and killing cancer cells $[16,17]$. The NDV is a paramyxovirus, round in shape with a bilayer lipid envelope [18]. Oncolysis mediated by NDV occurs through either direct cancer cell killing via the formation of syncytium or indirect cancer cell killing by stimulating an antitumour immune response [19]. To date, no studies have been conducted to determine the potential of CERS2 as a biomarker that can predict NDV oncolytic response in bladder cancer.

\section{Materials and Methods}

2.1. Cell Culture of Bladder Cell Lines. The seventeen bladder cell lines used in this study were purchased from the American Type Culture Collection (ATCC, Manassas, VA, USA) and the European Collection of Authenticated Cell Cultures (ECACC, Salisbury, UK). Cells were cultured and maintained with growth media based on the suppliers' recommendation. All the media were supplemented with fetal bovine serum (FBS) (Thermo Fisher Scientific Inc., Waltham, MA, USA). Cells were incubated at $37^{\circ} \mathrm{C}$ in a humidified atmosphere of $95 \%$ air and $5 \% \mathrm{CO}_{2}$.

2.2. Analysis of CERS2 Expression in a Panel of Bladder Cancer Cell Lines. RNeasy Mini Kit (Qiagen, Hilden, Germany) was used for extracting the RNA of harvested cells following the manufacturer's protocol. Quantification of the purity and concentration of the RNA was carried out using PCRmax Lambda spectrophotometer (PCRmax, Staffordshire UK). A total of $1 \mu \mathrm{g}$ RNA was converted to cDNA using QuantiTect ${ }^{\circledR}$ Reverse Transcription Kit (Qiagen, Hilden, Germany). The cDNA synthesis was performed using a thermocycler (Eppendorf, Hamburg, Germany) with the following conditions: incubation for 15 and 3 minutes at $42^{\circ} \mathrm{C}$ and $95^{\circ} \mathrm{C}$, respectively. The quantification of CERS2 expression levels was then carried out using a miScript SYBR Green PCR Kit (Qiagen, Hilden, Germany) on a LightCycler 480 (Roche, Basel, Switzerland). The qRT-
PCR assay was run in triplicates for 45 cycles with the following conditions: preincubation at $95^{\circ} \mathrm{C}$ and $94^{\circ} \mathrm{C}$ for 15 minutes and 15 seconds, respectively, followed by amplification for 45 cycles at $55^{\circ} \mathrm{C}, 70^{\circ} \mathrm{C}$, and $95^{\circ} \mathrm{C}$ for 15,30 , and 5 seconds, respectively. Subsequently, the melting phase analysis was conducted from $65^{\circ} \mathrm{C}$ for 1 minute, followed by a continuous increment to $97^{\circ} \mathrm{C}$ before finally cooling at $40^{\circ} \mathrm{C}$ for 30 seconds. The relative expression levels were determined by normalizing to $G A P D H, S D H A$, and $T B P$ housekeeping genes by using the $2^{-\triangle \Delta C T}$ method [20].

2.3. Knockdown of CERS2 Expression in UMUC1 Cells. Four small interfering RNA (siRNA) sequences that target CERS2 knockdown were purchased (Qiagen, Hilden, Germany). The sequences of the siRNAs were as follows: (1) HS_CERS2_5-5' AACCATCGTAAGAATGACTGA 3' (Cat. no. SI03124198), (2) HS_CERS2_6-5' CATGGC CGTCATTGTGGATAA 3' (Cat. no. SI04276671), (3) HS_CERS2_7-5' TGCGCTATAGGGTCACTTTAA 3' (Cat. no. SI04296684), and (4) HS_CERS2_8-5' CCGG CCCAGTCTCCTCAAGAA 3' (Cat. no. SI04711588). Nonsilencing siRNA with no homology to any known mammalian gene (Cat. no. SI03650318) was used as the negative control (Qiagen, Hilden, Germany) in all knockdown experiments. A total volume of $25 \mu \mathrm{L}$ of siRNA was added into each well according to the manufacturer's protocol, and the expression of CERS2 in UMUC1 was determined via qRTPCR at 24, 48, and 72 hours post-siRNA transfection.

2.4. Cell Migration Assay. The migratory potential of UMUC1 cells was measured using a scratch assay. Approximately 60,000 cells were seeded into a 24 -well plate. Once the cells reached $100 \%$ confluency, $10 \mathrm{~g} / \mathrm{mL}$ of mitomycin $\mathrm{C}$ (Merck, Darmstadt, Germany) was added to the cells and incubated for two hours to stop cell proliferation. A scratch was then made using a $200 \mu \mathrm{L}$ pipette tip, and the cells were washed with 1x PBS and replenished with fresh media. The cell migration progression was analysed under a microscope. Pictures were captured at three-hour intervals until the gap was closed. The pictures were analysed using the ImageJ software [21].

2.5. Immunohistochemical Analysis of CERS2 Protein Expression in Clinical Bladder Tissues. Sixty-two formalinfixed paraffin-embedded (FFPE) tissues from bladder cancer patients of various stages and grades were collected from Hospital Kuala Lumpur. The cohort of tissues comprised of 17 Grade 1, 18 Grade 2, and 27 Grade 3 tumours, of which, there were $18 \mathrm{pTa}, 19 \mathrm{pT} 1,11 \mathrm{pT} 2$, and $14 \mathrm{pT} 3$ and pT4 stages. Tissues were deparaffinized at $36^{\circ} \mathrm{C}$ for 1 hour. Slides were sequentially rehydrated with $100 \%, 95 \%, 80 \%$, and $70 \%$ ethanol immersions, followed by antigen retrieval which was done by boiling the slides in sodium citrate using a microwave. The sections were then incubated with a primary polyclonal rabbit antibody (ab85567) against CERS2 (Abcam, Cambridge, United Kingdom) at $4^{\circ} \mathrm{C}$ overnight and then incubated with Dako DAB chromogen (Dako, Glostrup, Denmark) secondary antibody at room temperature for 10 minutes. A negative control was run in every experiment by 


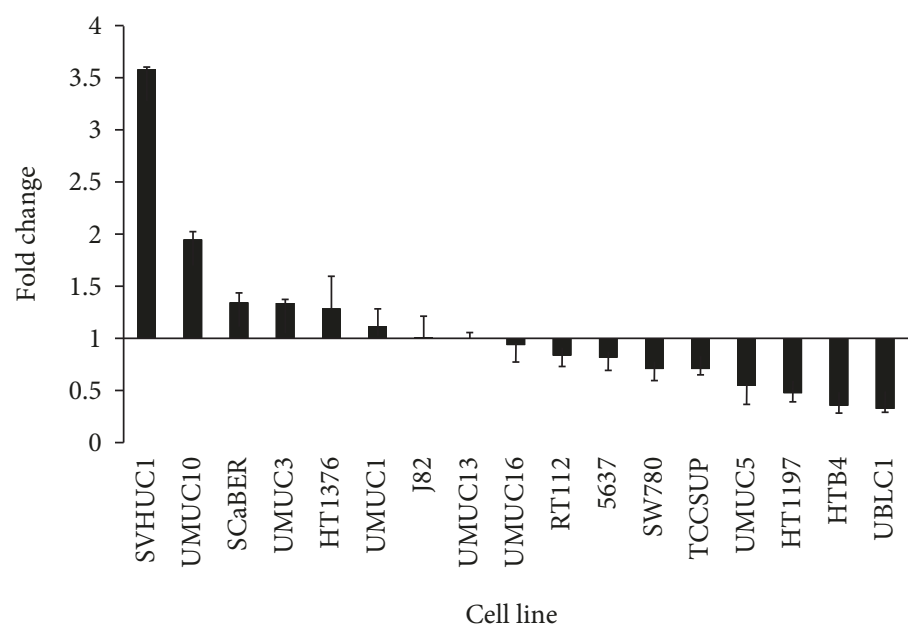

FIGURE 1: Expression of CERS2 across different bladder cell lines. The CERS2 gene expression levels in the 17 bladder cell lines as measured by qRT-PCR, ordered from the highest to lowest expression levels. The fold change for each cell line was calculated relative to the expression of CERS2 in UMUC13 cells after normalization with the expression levels of housekeeping genes.

replacing the primary antibody with $10 \%$ TBS. Sections were finally counterstained using haematoxylin and sequentially dehydrated with $70 \%, 80 \%, 95 \%$, and $100 \%$ ethanol immersions, mounted with DPX, and then observed under the microscope. Scoring was based on staining intensity.

2.6. Correlation between CERS2 Gene Expression and Sensitivity towards NDV-Mediated Oncolysis. The WST proliferation assay was carried out to determine the cell viability after infection with the NDV AF2240 strain at 5 different concentrations: $0,1,3,5$, and 7 HA units using a spectrophotometer at an absorbance of $440 \mathrm{~nm}$. The cell viability test was conducted on 14 cell lines (HTB4, UMUC5, UMUC10, UMUC1, UMUC3, UMUC13, UMUC16, TCCSUP, 5637, SCaBER, RT112, J82, SW780, and HT1376) and calculated at 4 different time points: 24, 48, 72, and 96 hours postinfection. The $\mathrm{IC}_{50}$ values were also determined at these four time points. The lower the $\mathrm{IC}_{50}$ values, the higher the degree of sensitivity towards NDV-mediated oncolysis. Correlation coefficient (Pearson correlation) analysis was then employed to find the correlation between the expression values and $\mathrm{IC}_{50}$ values. The data was analysed and expressed as the correlation coefficient, $R^{2}$ ( $R$-squared).

2.7. Statistical Analysis. The data in this study were expressed as mean \pm standard deviation. Independent $t$-test using GraphPad Prism Version 5.0.3 (GraphPad Inc., US) was employed to determine the significance of the quantitative data obtained. The Kruskal-Wallis test was employed to test the significance of expression of the CERS2 protein across different bladder cancer stages and grades. Results were significant if $p<0.05$.

\section{Results}

3.1. CERS2 In Vitro Gene Expression. The CERS2 gene expression levels of a panel of bladder cell lines were determined. The cell line with the median level of CERS2 expression, UMUC13, was selected as the reference cell line. The fold change of CERS2 expression relative to that of UMUC13 was determined for the remaining cell lines (Figure 1). The SVHUC1 and UMUC10 bladder cell lines were the highest CERS2-expressing cell lines with fold changes of 3.58 and 1.95, respectively. Incidentally, SVHUC1 is the only non-cancer cell line included in the study. The cell line was derived from the normal urothelium of the ureter and has been SV40 immortalised. Although it is derived from nonmalignant epithelial cells, $50 \%$ of its cells are tetraploid and they harbour various chromosomal aberrations. The lowest CERS2-expressing cell lines were UBLC1 and HTB4 with fold changes of 0.33 and 0.36 , respectively.

3.2. CERS2 Knockdown In Vitro. As compared to the negative control, the levels of the CERS2 gene were significantly reduced in cells transfected with the combination of 4 CERS2-targeting siRNAs. A significant reduction (47.5$67.5 \%)$ in the CERS2 expression was obtained at 24 and 48 hours posttransfection in CERS2 siRNA-transfected UMUC1 cells relative to the nontargeting siRNA-transfected negative control cells (Figure 2(a)). There was no significant reduction in the expression levels of CERS2 at 72 hours posttransfection. However, the effect of gene expression knockdown on the protein expression of CERS2 was not evaluated in vitro.

3.3. Effect of CERS2 Knockdown on Cell Migration. The scratch assay was employed to investigate the effect of CERS2 knockdown on UMUC1 cell motility. Images were captured at three-hour intervals from the time the scratch was made until the gap was closed for both CERS2 siRNA-transfected cells and nontargeting siRNA-transfected negative control cells (Figure 2(b)). The gap in the negative control cells closed within 30 hours, whereas the gap for the CERS2-knockdown cells closed within 42 hours postscratch. The rates of gap closure of CERS2-knockdown and negative control cells were significantly different $(p<0.05)$. 


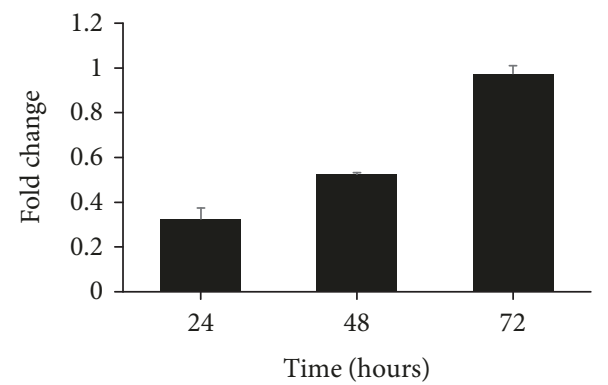

(a)
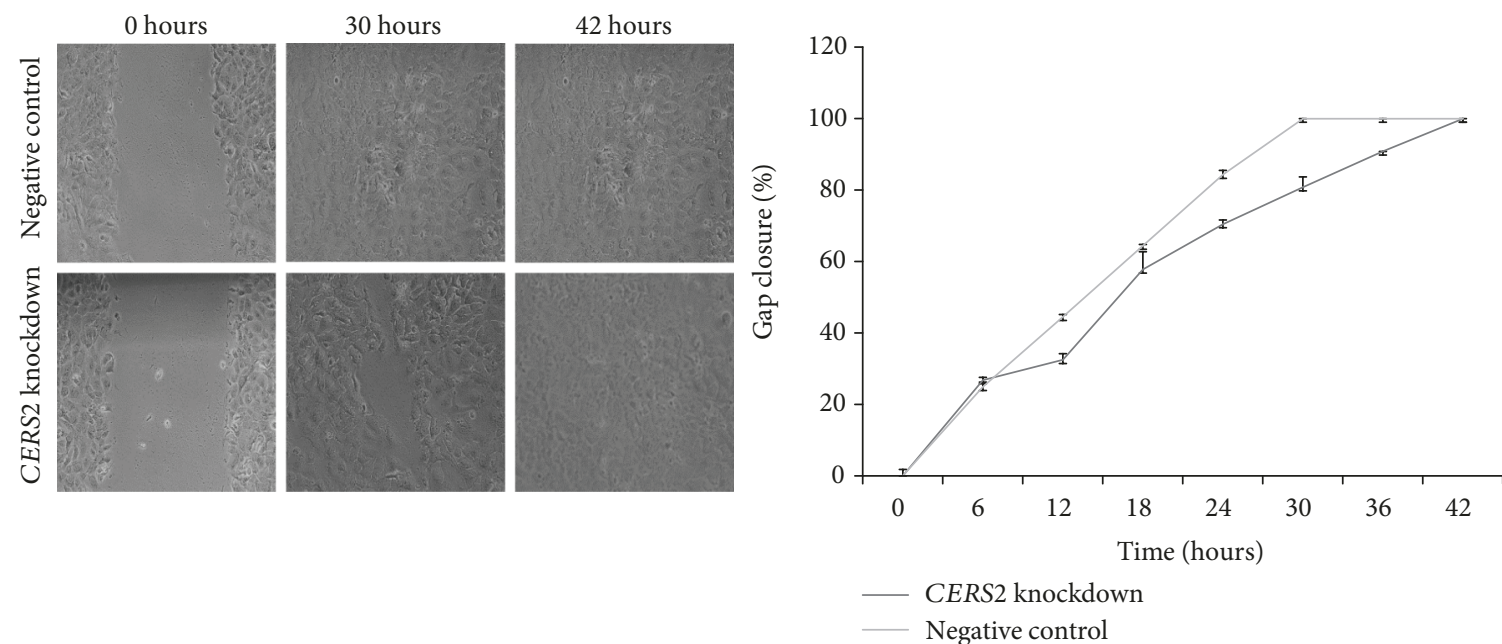

(b)

FIGURE 2: Effect of CERS2 knockdown on cellular migration in UMUC1 cells. (a) Fold change differences in the expression of CERS2 between siRNA-mediated CERS2-knockdown UMUC1 cells relative to the nontargeting siRNA-transfected negative control at 24, 48, and 72 hours posttransfection. (b) The percentage of gap closure of CERS2 knockdown versus negative control UMUC1 cells (200x magnification). CERS2-knockdown UMUC1 cells have significantly reduced migratory potential after 6 hours postscratch, corresponding to 30 hours post CERS2-siRNA transfection.

3.4. Analysis of CERS2 Protein Expression in Histopathological Tissues. Optimal CERS2 antibody concentration was determined by testing the various concentrations of primary antibodies. After a series of optimisation experiments, the optimal antibody dilution was determined to be $1: 250$. All the tissues were scored according to their staining intensities, categorised as weak, moderate, or strong staining (Figure 3). CERS2 staining was mostly localized in the cytoplasm. The majority of Grade 1 tissue samples displayed weak staining intensity, whereas a greater proportion of Grade 2 and 3 tumours had a stronger staining intensity. Interestingly, while a greater proportion of Stage 4 tumours expressed high levels of CERS2 as compared to other tumour stages, there was also a comparatively greater percentage of Stage 4 tumours that expressed low levels of CERS2. This was because there were a smaller proportion of Stage 4 tumours that displayed moderate expression of CERS2. Nevertheless, no statistical significant differences in CERS2 protein expression across different tumour stages and grades were identified in this study (Figures 3(e) and 3(f)).

3.5. Correlation Analysis between CERS2 Gene Expression and NDV-Mediated Oncolysis. The correlation analysis was done to determine whether the expression of CERS2 was associated with bladder cancer cellular sensitivity towards NDV-mediated oncolysis. The correlation coefficient $R^{2}$ values were 0.0383 at 24 hours postinfection $(r=0.19$; Figure $4(\mathrm{a})), 0.0077$ at 48 hours postinfection $(r=0.087$; Figure 4(b)), 0.0058 at 72 hours postinfection $(r=0.076$; Figure $4(\mathrm{c}))$, and 0.0055 at 96 hours postinfection $(r=0.074$; Figure $4(\mathrm{~d})$ ). All of the $R^{2}$ values obtained indicate that a weak correlation exists between the $\mathrm{IC}_{50}$ values and CERS2 gene expression levels across the four different time points.

\section{Discussion}

The CERS2 gene was found to be differentially expressed across the different bladder cancer cell lines that were investigated in this study. A study conducted by Zhao et al. [22] found that the lower the expression levels of CERS2, the more aggressive the bladder cancer cell lines were, suggesting that CERS2 expression correlates with bladder cancer progression and metastasis. The cell lines used in this study were ranked based on the level of CERS2 expression and mapped to the histology, molecular subtype, genetic instability, and mutational status of classical genes associated with bladder cancer 


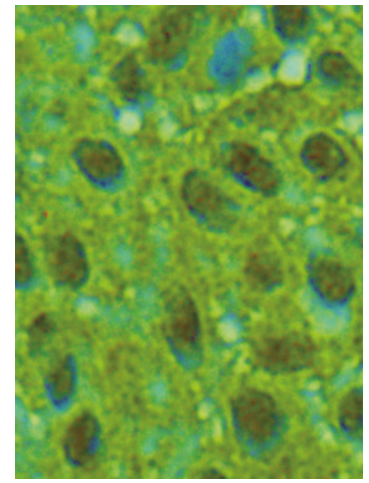

(a)

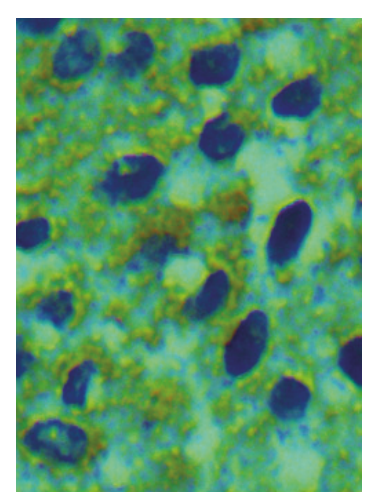

(b)

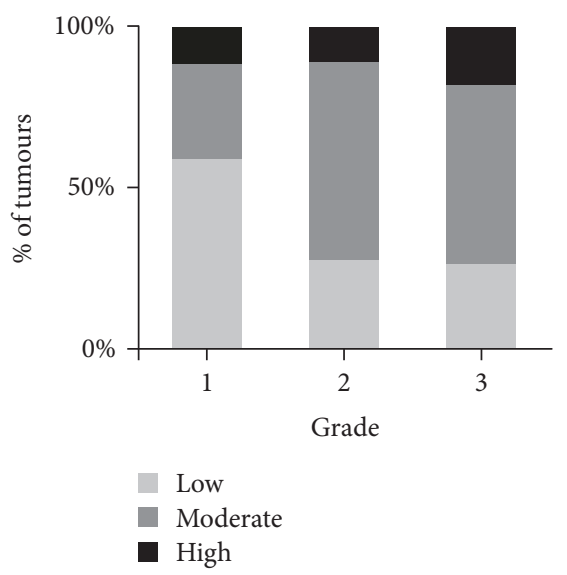

(e)

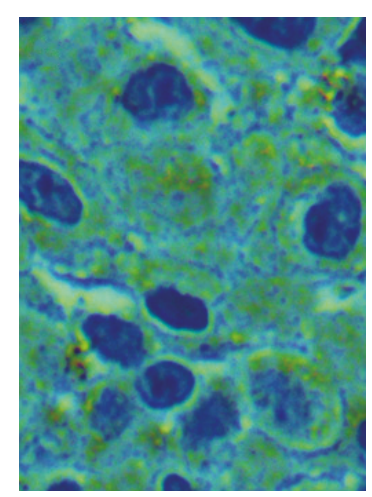

(c)

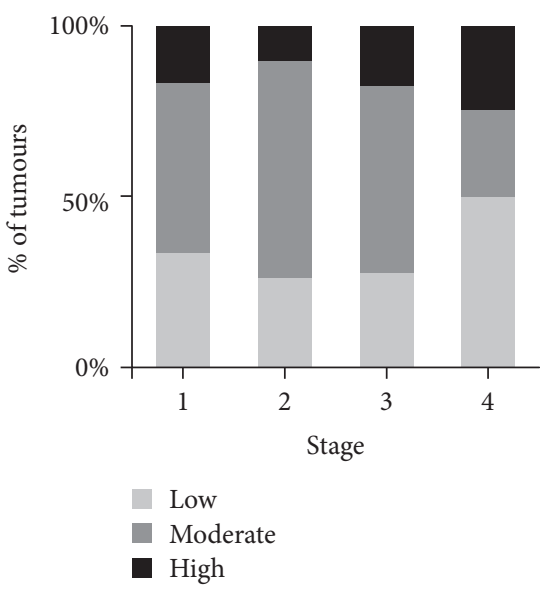

(f)

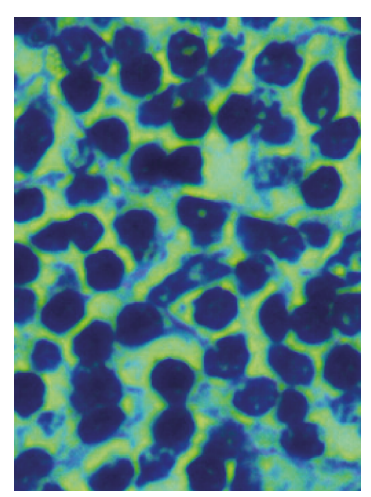

(d)

FIGURE 3: CERS2 protein staining in clinical bladder cancer tissues. Representative cases of (a) strong staining (high expression), (b) moderate staining (moderate expression), (c) weak staining (low expression), and (d) negative control at 400x magnification. The expression of CERS2 in the tissue samples was evaluated based on the staining intensity. CERS2 protein expression in bladder cancer tissues stratified based on tumour grade (e) and stage (f).

as determined and catalogued in previous studies [23-25] (Figure 5). The only squamous cell carcinoma cell line in this study, SCaBER, was the $2^{\text {nd }}$ highest CERS2-expressing cell line. There were no obvious distinctions between high- and low-CERS2-expressing cell lines in terms of the grade of the primary tumour, molecular subtype (basal, mixed, and luminal) and genetic stability. Interestingly, the five highest CERS2-expressing cell lines harboured lower frequency of FGFR3 and PIK3CA mutations as compared to the lower CERS2-expressing cell lines. The differential CERS2 expression across the various bladder cancer cell lines in this study reflects tumour heterogeneity and that the functional significance of CERS2 expression may vary across different bladder tumours.

To investigate the metastasis-suppressing potential function of CERS2, the expression of CERS2 was downregulated through siRNA-mediated knockdown. CERS2 knockdown was established in UMUC1 cells instead of the higher CERS2-expressing bladder cancer cell lines (UMUC10, SCaBER, UMUC3, and HT1376) because these cells were growing at a very slow rate. The CERS2 expression was downregulated by $47.5 \%$ at 24 hours and $67.5 \%$ at 48 hours posttransfection. The downregulation of CERS2 expression reduced the migratory potential of UMUC1 cells. These findings are in contrast to the findings by Wang et al. [26], where they found that the migratory potential of RT4 and T24 bladder cancer cell lines increased upon siRNAmediated knockdown of CERS2 expression. The opposing roles that CERS2 plays in different types of bladder cancer cells suggest that the influence of CERS2 on the migratory potential phenotype is dependent on other factors such as the combinatorial expression of other genes.

For instance, although CERS2 is involved in the production of ceramide that in turn inhibits the migration, proliferation, and survival of cancer cells [27], ceramide can be metabolized to form sphingosine 1-phosphate (S1P), an antiapoptotic agent that increases cancer cell migration and proliferation as well as survival [28]. The UMUC1 cells that express CERS2 potentially produce ceramide that is subsequently metabolized into S1P. Thus, the knockdown of CERS2 may have led to the lowering of ceramide levels, resulting in the reduction of S1P production that in turn caused the inhibition of the migratory potential of the UMUC1 CERS2-knockdown cells. Another reason for the decreased migratory potential in the CERS2-knockdown cells could be explained through another study in neuroblastoma 


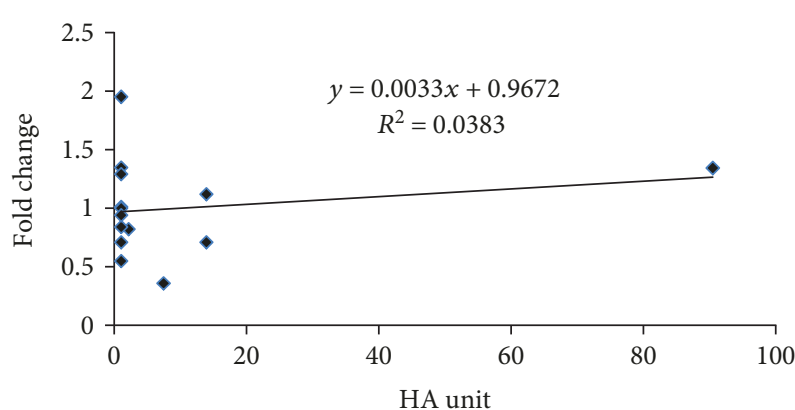

(a)

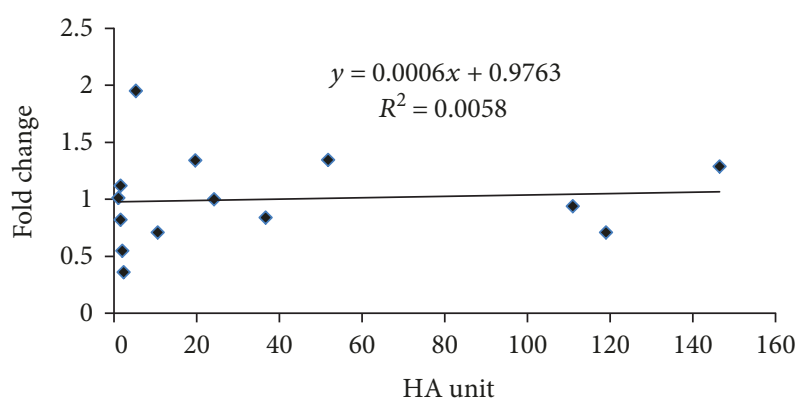

(c)

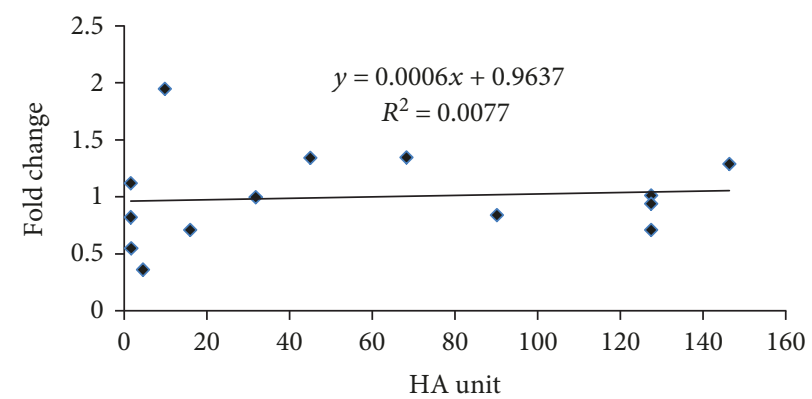

(b)

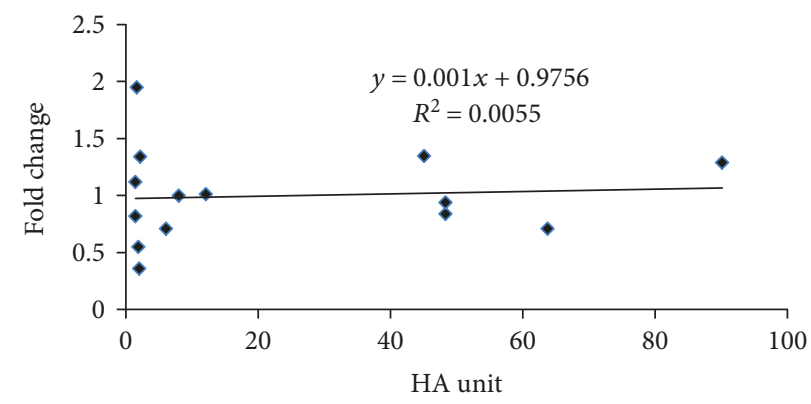

(d)

FIGURE 4: Correlation between CERS2 gene expression and sensitivity to NDV-mediated oncolysis. The series of graphs show the correlation between $\mathrm{IC}_{50}$ values and CERS2 expression across 14 cell lines at (a) 24 hours, (b) 48 hours, (c) 72 hours, and (d) 96 hours post-NDV infection. The correlation efficiencies are presented as $R^{2}$ values.

[29]. Findings from the study suggest that CERS2 knockdown activates the unfolded protein response and induces autophagy, thus decreasing the migratory rate of transfected cells as observed in our study.

In this study, the mechanism in which CERS2 knockdown exerts an antimigratory effect on UMUC1 bladder cancer cells is not established, but while many studies suggest CERS2 as a putative pan-cancer metastatic suppressor gene, there are studies that suggest a more protumourigenic role for CERS2, albeit to a lesser extent $[12,30]$. The study conducted on breast cancer tissues by Schiffmann et al. [12] revealed a 12 -fold increase in the levels of CERS2 in malignant tissues as compared to normal tissues, while the study conducted by Erez-Roman et al. [30] showed that CERS2 was highly expressed in about half of the invasive ductal carcinoma patients examined. The duality of genes that harbour functions as oncogenes and tumour suppressor genes, manifesting differently in different tumours, have been identified in many other protumourigenic genes with tumour-suppressive function ([31]). Thus, further studies need to be conducted to effectively delineate the context in which the level of CERS2 affects bladder cancer cell phenotype.

To investigate the potential role of CERS2 as a biomarker for bladder cancer, immunohistochemistry analysis was conducted to evaluate the patterns of expression in bladder cancer histopathological tissues. Although a greater proportion of high grade and stage tumours expressed high levels of CERS2 protein, there were no statistically significant differences in the expression levels across different grades and stages. This is contradictory to the findings from a previous study conducted by Wang et al. [11] that observed an association with the depth of invasion. A possible explanation for such discrepancy may be due to the small number of tissue samples screened in this study or the heterogeneous nature of the disease.

Previous studies have demonstrated the oncolytic activity of the NDV AF2240 strain as an anticancer agent against cancer cells [32]. An inverse correlation between antiviral gene expression and NDV susceptibility in several cell types has been identified [33]. This study found a weak correlation between CERS2 expression and $\mathrm{IC}_{50}$ values of NDV-mediated oncolysis. The weak correlation suggests that CERS2 expression is not a suitable biomarker for predicting bladder cancer response towards NDV anticancer therapy. However, further investigation into the interactions between the CERS2 gene and other genes within pathways related to the responses to NDV therapy may provide insights into identifying more promising biomarkers.

\section{Conclusion}

Findings from our study revealed that the CERS2 gene is differentially expressed in different types of bladder cancer cell lines. It was demonstrated that the knockdown of the CERS2 gene resulted in the reduction of the migratory potential of UMUC1 cells. The CERS2 protein was differentially expressed in the bladder cancer tissues analysed, where some tumours expressed high levels while some tumours expressed none or low levels of CERS2. However, there was no statistically significant association between the grade or stage and 


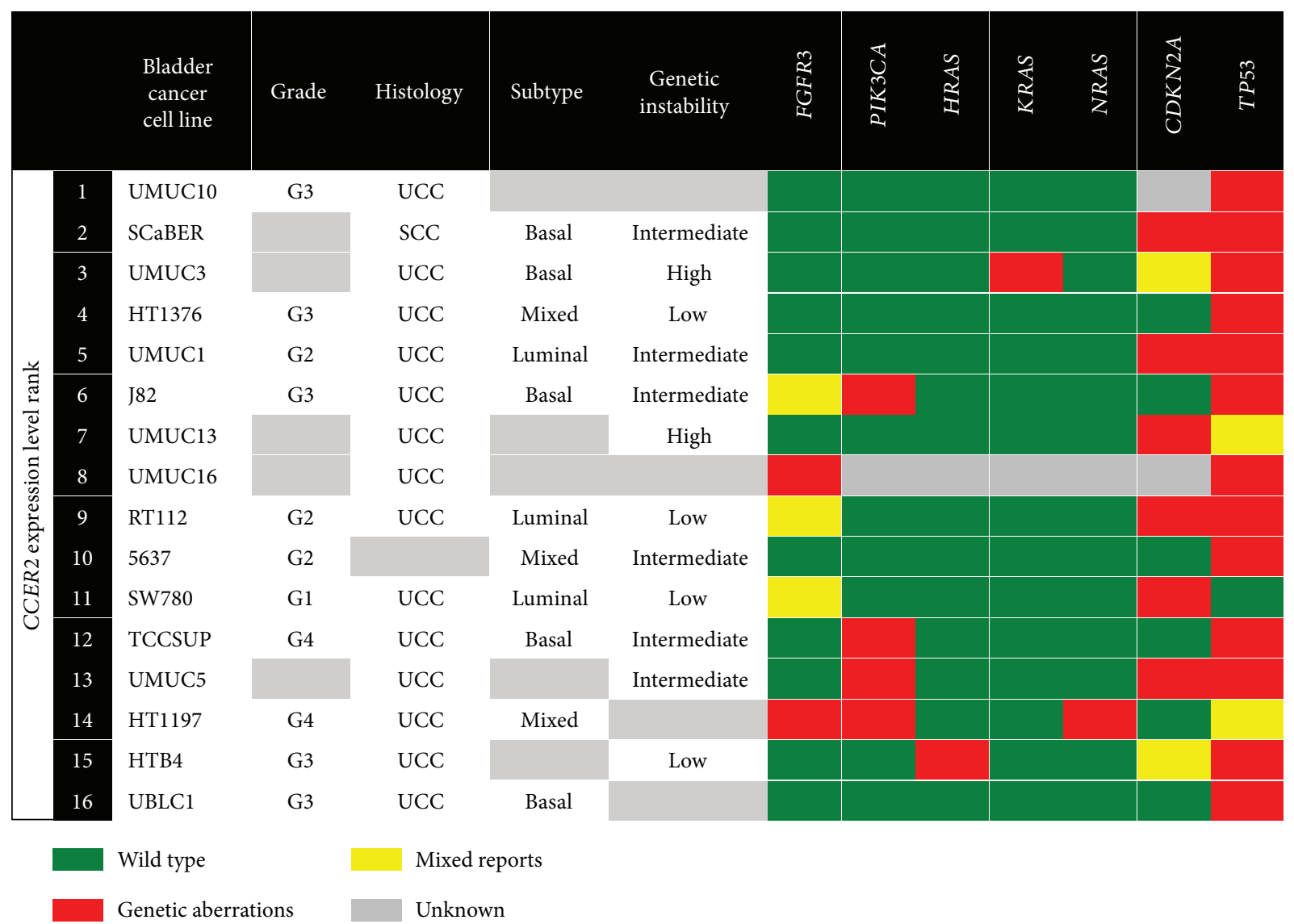

FIGURE 5: Molecular and histological characteristics of bladder cancer cell lines ranked based on CERS2 expression levels (highest expression (rank 1) to lowest expression (rank 16)). Source of molecular and histological characteristics: [23-25].

the intensity of staining. Finally, there was no correlation between the expression of CERS2 in bladder cancer cell lines and their sensitivity towards NDV-mediated oncolysis. The promigratory role of CERS2 identified in this study that contrasts previous studies in different bladder cancer cells should be further investigated to clarify the context in which the expression of CERS2 modulates the cancer cell migration phenotype.

\section{Data Availability}

The data used to support the findings of this study are available from the corresponding author upon request.

\section{Conflicts of Interest}

The authors declare that they have no conflicts of interest.

\section{Acknowledgments}

This study was supported by the University of Malaya High Impact Research (HIR) Grant Scheme UM.C/625/1/HIR/130 and the Ministry of Energy, Science, Technology, Environment and Climate Change Malaysia Flagship Fund, reference number FP0514B0021-2 (DSTIN).

\section{References}

[1] S. Antoni, J. Ferlay, I. Soerjomataram, A. Znaor, A. Jemal, and F. Bray, "Bladder cancer incidence and mortality: a global overview and recent trends," European Urology, vol. 71, no. 1, pp. 96-108, 2017.

[2] A. Jemal, R. Siegel, E. Ward et al., "Cancer statistics, 2008," CA: A Cancer Journal for Clinicians, vol. 58, no. 2, pp. 71-96, 2008.

[3] F. Darwiche, D. J. Parekh, and M. L. Gonzalgo, "Biomarkers for non-muscle invasive bladder cancer: current tests and future promise," Indian Journal of Urology, vol. 31, no. 4, pp. 273-282, 2015.

[4] Z. Kirkali, T. Chan, M. Manoharan et al., "Bladder cancer: epidemiology, staging and grading, and diagnosis," Urology, vol. 66, no. 6, pp. 4-34, 2005.

[5] S. van den Bosch and J. A. Witjes, "Long-term cancer-specific survival in patients with high-risk, non-muscle-invasive bladder cancer and tumour progression: a systematic review," European Urology, vol. 60, no. 3, pp. 493-500, 2011.

[6] R. Sawhney, D. Bourgeois, and U. B. Chaudhary, "Neoadjuvant chemotherapy for muscle-invasive bladder cancer: a look ahead," Annals of Oncology, vol. 17, no. 9, pp. 1360-1369, 2006.

[7] Z. Andreu, R. Otta Oshiro, A. Redruello et al., "Extracellular vesicles as a source for non-invasive biomarkers in bladder cancer progression," European Journal of Pharmaceutical Sciences, vol. 98, pp. 70-79, 2017. 
[8] A. Stenzl, N. C. Cowan, M. de Santis et al., "Treatment of muscle-invasive and metastatic bladder cancer: update of the EAU guidelines," European Urology, vol. 59, no. 6, pp. 1009-1018, 2011.

[9] X. F. Cai, Z. Tao, Z. Q. Yan, S. L. Yang, and Y. Gong, "Molecular cloning, characterisation and tissue-specific expression of human LAG3, a member of the novel Lag1 protein family," DNA Sequence, vol. 14, no. 2, pp. 79-86, 2003.

[10] C. Ma, Y. Liu, J. Zheng et al., "Identification of tumor metastasis-related gene TMSG-1 by mRNA differential display," Science in China Series C: Life Sciences, vol. 45, no. 5, pp. 553-560, 2002.

[11] H. Wang, J. Wang, Y. Zuo et al., "Expression and prognostic significance of a new tumor metastasis suppressor gene LASS2 in human bladder carcinoma," Medical Oncology, vol. 29, no. 3, pp. 1921-1927, 2012.

[12] S. Schiffmann, J. Sandner, K. Birod et al., "Ceramide synthases and ceramide levels are increased in breast cancer tissue," Carcinogenesis, vol. 30, no. 5, pp. 745-752, 2009.

[13] E. K. Vladar, Y. L. Lee, T. Stearns, and J. D. Axelrod, "Observing planar cell polarity in multiciliated mouse airway epithelial cells," Methods in Cell Biology, vol. 127, pp. 37-54, 2015.

[14] X. Y. Xu, F. Pei, and J. F. You, "TMSG-1 and its roles in tumor biology," Chinese Journal of Cancer, vol. 29, no. 7, pp. 697-702, 2010.

[15] X. Xu, J. You, and F. Pei, "Silencing of a novel tumor metastasis suppressor gene LASS2/TMSG1 promotes invasion of prostate cancer cell in vitro through increase of vacuolar ATPase activity," Journal of Cellular Biochemistry, vol. 113, no. 7, pp. 2356-2363, 2012.

[16] J. J. Nemunaitis, "Cancer treatment involving oncolytic viruses," Clinical Lung Cancer, vol. 1, no. 1, pp. 50-55, 1999.

[17] O. V. Matveeva, Z. S. Guo, V. M. Senin, A. V. Senina, S. A. Shabalina, and P. M. Chumakov, "Oncolysis by paramyxoviruses: preclinical and clinical studies," Molecular Therapy-Oncolytics, vol. 2, p. 15017, 2015.

[18] V. Schirrmacher and P. Fournier, "Newcastle disease virus: a promising vector for viral therapy, immune therapy, and gene therapy of cancer," Methods in Molecular Biology, vol. 542, pp. 565-605, 2009.

[19] O. V. Matveeva, Z. S. Guo, S. A. Shabalina, and P. M. Chumakov, "Oncolysis by paramyxoviruses: multiple mechanisms contribute to therapeutic efficiency," Molecular TherapyOncolytics, vol. 2, p. 15011, 2015.

[20] K. J. Livak and T. D. Schmittgen, "Analysis of relative gene expression data using real-time quantitative PCR and the $2^{-\Delta \Delta \mathrm{C}}$ method," Methods, vol. 25, no. 4, pp. 402-408, 2001.

[21] C. A. Schneider, W. S. Rasband, and K. W. Eliceiri, "NIH image to ImageJ: 25 years of image analysis," Nature Methods, vol. 9, no. 7, pp. 671-675, 2012.

[22] Q. Zhao, H. Wang, M. Yang, D. Yang, Y. Zuo, and J. Wang, "Expression of a tumor-associated gene, LASS2, in the human bladder carcinoma cell lines BIU-87, T24, EJ and EJ-M3," Experimental and Therapeutic Medicine, vol. 5, no. 3, pp. 942-946, 2013.

[23] J. Earl, D. Rico, E. Carrillo-de-Santa-Pau et al., "The UBC-40 urothelial bladder cancer cell line index: a genomic resource for functional studies," BMC Genomics, vol. 16, no. 1, p. 403, 2015.
[24] M. L. Nickerson, N. Witte, K. M. Im et al., "Molecular analysis of urothelial cancer cell lines for modeling tumor biology and drug response," Oncogene, vol. 36, no. 1, pp. 35-46, 2017.

[25] T. C. M. Zuiverloon, F. C. de Jong, J. C. Costello, and D. Theodorescu, "Systematic review: characteristics and preclinical uses of bladder cancer cell lines," Bladder Cancer, vol. 4, no. 2, pp. 169-183, 2018.

[26] H. Wang, Y. Zuo, M. Ding et al., "LASS2 inhibits growth and invasion of bladder cancer by regulating ATPase activity," Oncology Letters, vol. 13, no. 2, pp. 661-668, 2017.

[27] E. L. Laviad, L. Albee, I. Pankova-Kholmyansky et al., "Characterization of ceramide synthase 2 tissue distribution, substrate specificity, and inhibition by sphingosine 1-phosphate," Journal of Biological Chemistry, vol. 283, no. 9, pp. 5677-5684, 2008.

[28] B. Ogretmen, "Sphingolipid metabolism in cancer signalling and therapy," Nature Reviews Cancer, vol. 18, no. 1, pp. 33-50, 2018.

[29] S. D. Spassieva, T. D. Mullen, D. M. Townsend, and L. M. Obeid, "Disruption of ceramide synthesis by CerS2 downregulation leads to autophagy and the unfolded protein response," Biochemical Journal, vol. 424, no. 2, pp. 273-283, 2009.

[30] R. Erez-Roman, R. Pienik, and A. H. Futerman, "Increased ceramide synthase 2 and 6 mRNA levels in breast cancer tissues and correlation with sphingosine kinase expression," Biochemical and Biophysical Research Communications, vol. 391, no. 1, pp. 219-223, 2010.

[31] L. Shen, Q. Shi, and W. Wang, "Double agents: genes with both oncogenic and tumor-suppressor functions," Oncogene, vol. 7, no. 3, p. 25, 2018.

[32] U. Ahmad, I. Ahmed, Y. Y. Keong, N. Abd Manan, and F. Othman, "Inhibitory and apoptosis-inducing effects of Newcastle disease virus strain AF2240 on mammary carcinoma cell line," BioMed Research International, vol. 2015, Article ID 127828, 12 pages, 2015.

[33] H. Wilden, P. Fournier, R. Zawatzky, and V. Schirrmacher, "Expression of RIG-I, IRF3, IFN- $\beta$ and IRF7 determines resistance or susceptibility of cells to infection by Newcastle disease virus," International Journal of Oncology, vol. 34, no. 4, pp. 971-982, 2009. 


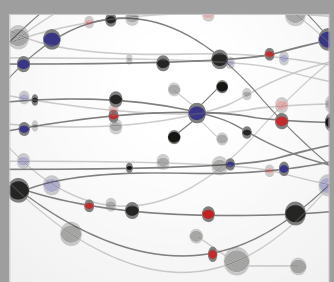

The Scientific World Journal
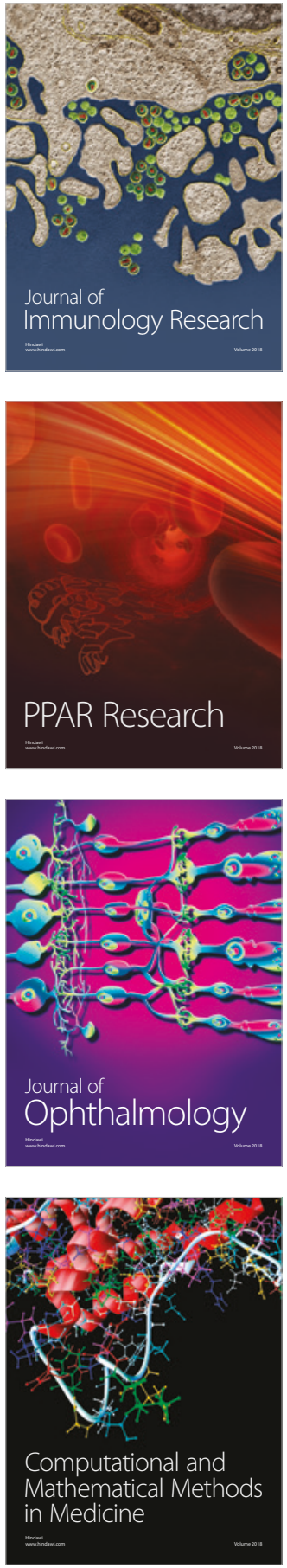

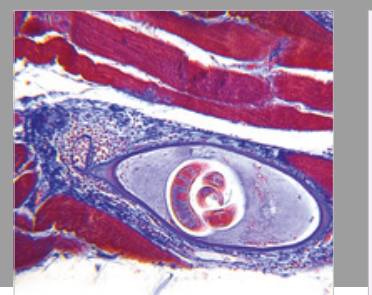

Gastroenterology Research and Practice

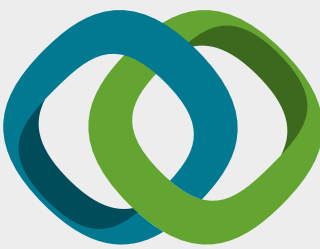

\section{Hindawi}

Submit your manuscripts at

www.hindawi.com
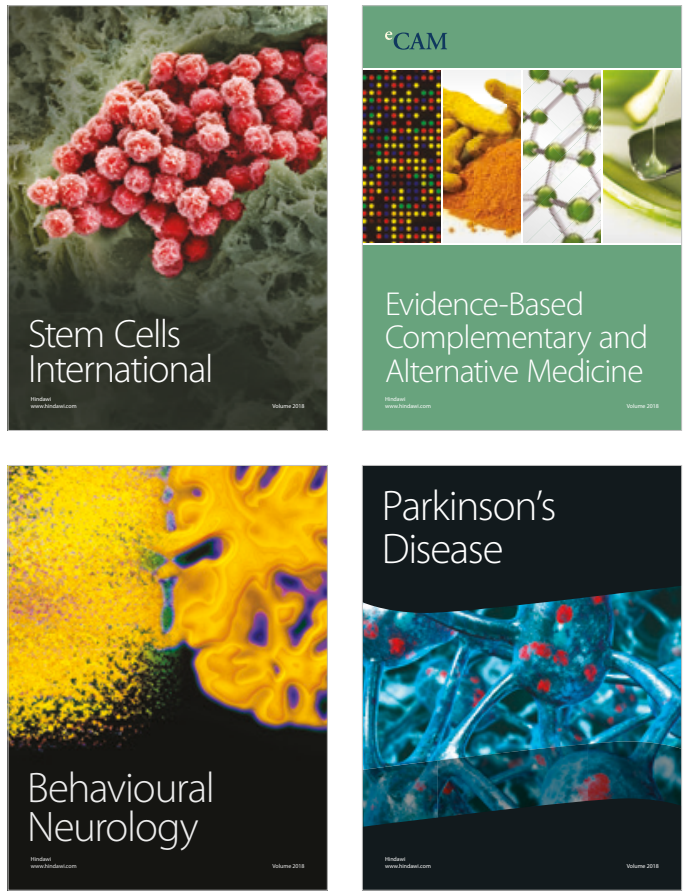

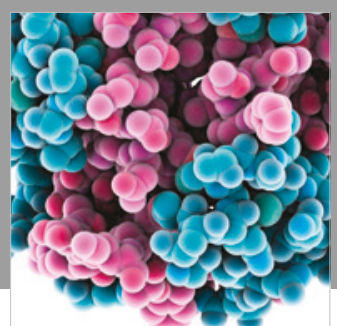

ournal of

Diabetes Research

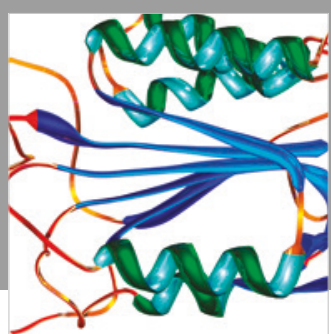

Disease Markers
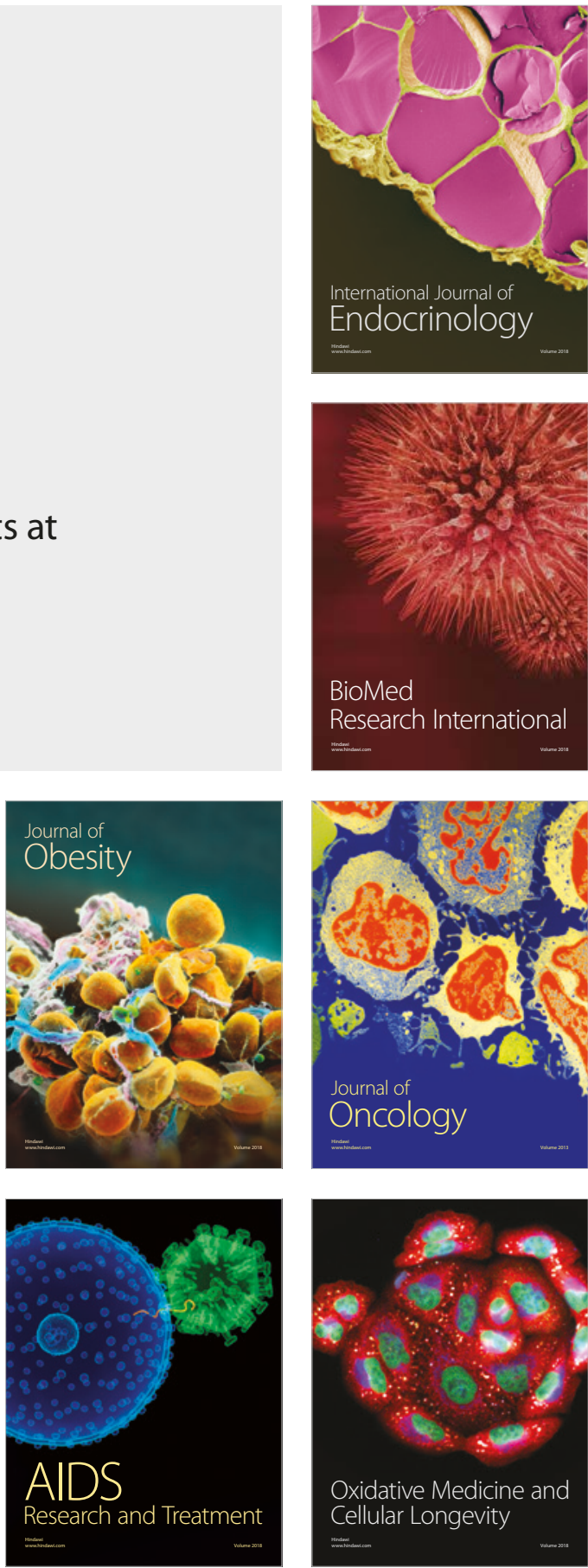